\title{
No Giant Planet Pileup Near 1 AU
}

\author{
A. Wise and S. Dodson-Robinson \\ University of Delaware
}

\section{Introduction}

Using semimajor axis distributions with logarithmic spacing, many investigators (Udry \& Santos 2007; Wright et al.|2009; Hasegawa \& Pudritz|2012; Beaugé \& Nesvorný|2013) have suggested that there exists a pileup (or other distribution feature near $1 \mathrm{AU}$, which we hereafter refer to as a pileup) in the distribution of giant exoplanets near 1-AU. We propose that the pileup (or any distribution feature) is not significant. Since we do not have knowledge of individual RV surveys' many complex sampling and selection biases, we cannot assign a proper statistical significance to the pileup. Given access to the publicly available data only, analyses akin to the following may be the best way to assess whether the claimed pileup exists in the current set of confirmed exoplanets.

The mass-semimajor axis distribution of RV-detected giant planet:1 is shown on a logarithmic semimajor axis scale (top-left), and a linear scale (top-right) truncated at 2.5 AU to avoid observational biases against detecting long-period planets. From these plots, it is clear that giant planet semimajor axes are not log-uniformly distributed, a fact that partly triggered the pileup proposal. However, we assert that the expected exoplanet semimajor axis distribution should not be log-uniform. A simple argument is as follows: When looking for non-uniform features in the distribution of $\log (a)$, the null hypothesis is that the giant planet frequency scales with $1 / a$, as the plot interval allotted to each AU of semimajor axis goes as 1/a. According to Armitage (2007), Bitsch \& Kley (2011a b), and Coleman \& Nelson (2014), the majority of giant planets within 5 AU probably underwent significant inward migration after forming. If they migrated at local viscous timescale, $r^{2} / \nu$, and we assume $\nu(r) \sim r$ (e.g. Hartmann et al. 1998), these planets would migrate slower at larger $a$, so giant planet frequency per AU would increase with $a$.

\section{Bayesian Blocks Analysis}

Here we use the Bayesian Blocks algorithm (Scargle et al. 2013) to assess the extent to which the 1-D distribution of giant planet semimajor axes, plotted on a linear scale, differs from a uniform distribution. First we resample the exoplanet mass-semimajor axis distribution using reported observational uncertainties ${ }^{1}$. For each planet and each variable (mass, semimajor axis), we construct two Gaussian probability density functions (PDFs), one from the upper and one from the lower 1- $\sigma$ error. We randomly select which PDF to use and then pick a random value from its domain (with

\footnotetext{
${ }^{1}$ Planet orbit and mass data from exoplanet.eu/catalogs, March 16, 2017 (Schneider et al. 2011)
} 
probability weighted by PDF), adding this error value to the reported semimajor axis or mass. For reported errors that were undefined or zero according to exoplanet.eu, we assumed 1- $\sigma$ errors of $1 \%$ of the planet's reported mass or semimajor axis - much tighter than typical reported error bar measurements (Schneider et al. 2011). We resampled the mass-semimajor axis distribution 1000 times, then computed each corresponding 1-D semimajor axis distribution for planets with resampled $M \sin i>0.5 M_{\text {Jup }}$. The ensemble of $10001-\mathrm{D}$ semimajor axis distributions for giant planets forms the solid blue histogram shown in the middle-left.

Now, we use the Bayesian Blocks algorithm to construct optimally binned semimajor axis histograms of giant exoplanets (Scargle et al. 2013): We adopt a geometric prior on the number of blocks (histogram bins), $P\left(N_{\text {blocks }}\right)=P_{0} \gamma^{N_{\text {blocks }}}$, and choose a threshold probability for correct detection of all bin edges ("change points") of $p_{*}=0.95$. For each resampled semimajor axis distribution A containing $\mathrm{N}_{\mathrm{A}}$ planets, we compute an appropriate $\gamma$ using a set of random datasets $\mathrm{R}_{\mathrm{N}_{\mathrm{A}}}$ (all with $\mathrm{N}_{\mathrm{A}}$ planets) drawn from a uniform semimajor axis distribution. First, for each of a large number of $R_{N_{A}}$, we find the largest $\gamma$ for which the Bayesian Blocks algorithm applied to $\mathrm{R}_{\mathrm{N}_{\mathrm{A}}}$ finds a number of change points less than or equal to $\left(1-p_{*}\right)\left(\mathrm{N}_{\mathrm{A}}-1\right)$, and average over these values of $\gamma$. Second, we use this average $\gamma$ in our prior as we use the Bayesian Blocks algorithm to find the number of change points in $\mathrm{A}, N_{c p}$. These two steps are repeated, only changing the maximum number of change points detected to $\left(1-p_{*}^{1 / N_{c p}}\right)\left(\mathrm{N}_{\mathrm{A}}-1\right)$ using the value of $N_{c p}$ from each previous iteration, until $N_{c p}$ stops changing. This iterative algorithm does not converge if a dataset A is truly uniform, with $N_{c p}=0$, and we note 83 out of 1000 resampled exoplanet semimajor axis distributions did not converge within $>100$ iterations, so we treat these 83 distributions as having zero change points. The two bottom plots show sample semimajor axis histograms produced by the Bayesian Blocks algorithm.

The middle-right shows a histogram of the histogram change-point locations from our Bayesian Blocks analysis. The most notable feature is $78 \%$ of resampled realizations of planet semimajor axes show higher planet occurrence rate between 1 and 1.5 AU, indicated by a pair of change points - one to increase the planet frequency (positive in plot), and a second to decrease it (negative in plot). As this feature only appears in $78 \%$ of resampled semimajor axis distributions, we suggest that the evidence for a pileup near $1 \mathrm{AU}$ is not significant.

The authors thank Eric Ford for useful ideas and acknowledge support from the UNIDEL foundation and NSF CAREER award 1520101. 

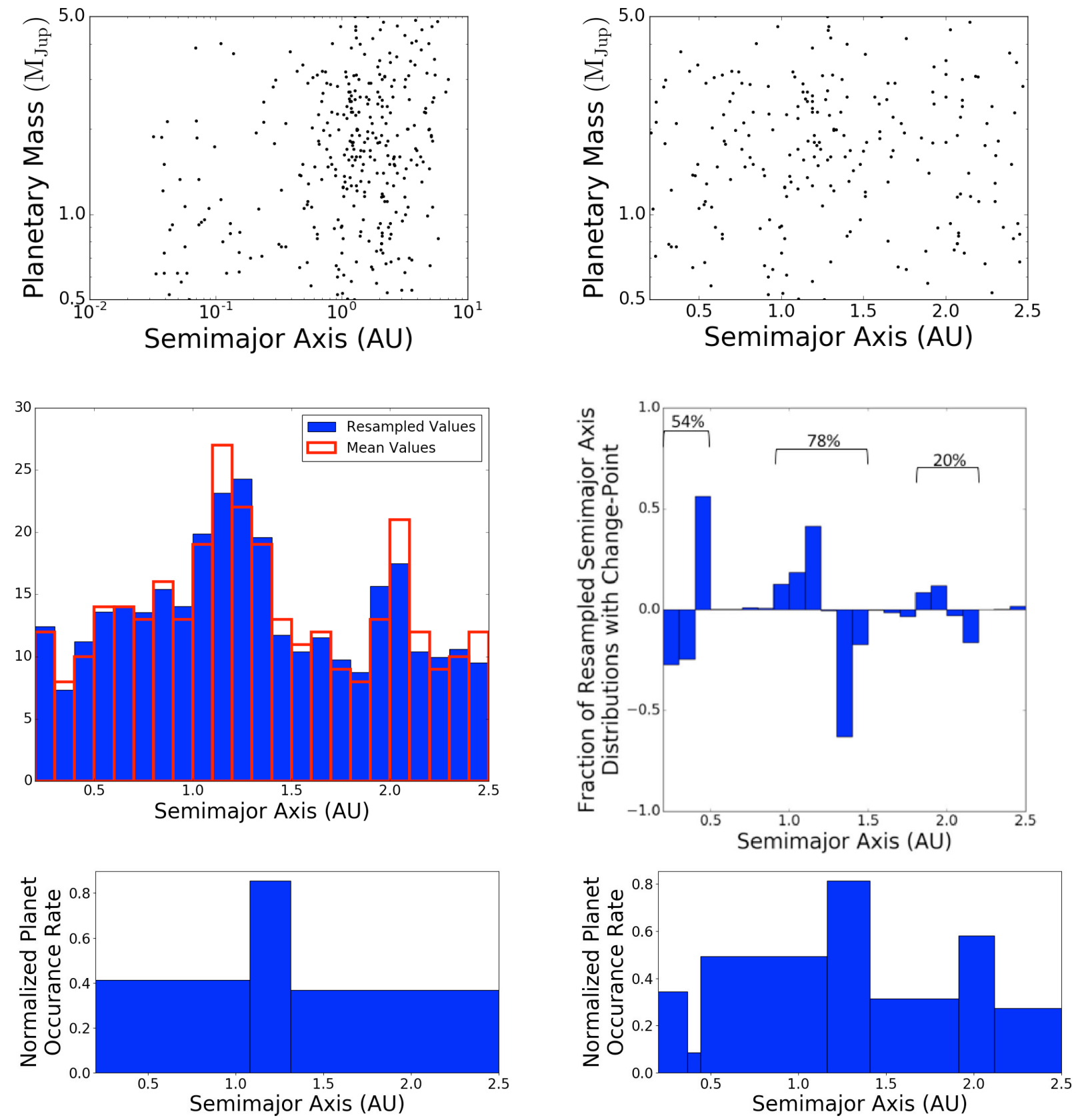

Figure: Plots showing the results of our analysis of the semimajor axis distribution of giant exoplanets detected by RV measurements. See text for plot descriptions. 


\section{REFERENCES}

Armitage, P. J. 2007, ApJ, 665, 1381

Beaugé, C., \& Nesvorný, D. 2013, ApJ, 763, 12

Bitsch, B., \& Kley, W. 2011a, A\&A, 530, A41

-. 2011b, A\&A, 536, A77

Coleman, G. A. L., \& Nelson, R. P. 2014, MNRAS, 445, 479

Hartmann, L., Calvet, N., Gullbring, E., \& D’Alessio, P. 1998, ApJ, 495, 385

Hasegawa, Y., \& Pudritz, R. E. 2012, ApJ, 760, 117

Scargle, J. D., Norris, J. P., Jackson, B., \& Chiang, J. 2013, ApJ, 764, 167

Schneider, J., Dedieu, C., Le Sidaner, P., Savalle, R., \& Zolotukhin, I. 2011, A\&A, 532, A79

Udry, S., \& Santos, N. C. 2007, ARA\&A, 45, 397

Wright, J. T., Upadhyay, S., Marcy, G. W., et al. 2009, ApJ, 693, 1084 